\title{
Do negative emotions expressed during follow-up consultations with adolescent survivors of childhood cancer reflect late effects?
}

\author{
Anneli V. Mellblom, ${ }^{1,2}$ Ellen Ruud, ${ }^{2,3}$ Jon Håvard Loge, ${ }^{1,4}$ \& Hanne C. Lie $e^{1,2,5}$ \\ ${ }^{1}$ Department of Behavioural Sciences in Medicine, Institute of Basic Medical \\ Sciences, Faculty of Medicine, University of Oslo, Oslo, Norway. \\ ${ }^{2}$ Department of Pediatric Medicine, Women and Children's Unit, Oslo University \\ Hospital, Rikshospitalet, Oslo, Norway. \\ ${ }^{3}$ Institute of Clinical Medicine, Faculty of Medicine, University of Oslo, Norway. \\ ${ }^{4}$ Regional Advisory Unit for Palliative Care, Dept. of Oncology, Oslo University \\ Hospital (OUS), Oslo, Norway. \\ ${ }^{5}$ National Resource Centre for Late Effects after Cancer Treatment, Oslo University \\ Hospital, Radiumhospitalet, Oslo, Norway. \\ Email addresses of authors: \\ Anneli Mellblom: anneli.mellblom@medisin.uio.no, Jon Håvard Loge: \\ j.h.loge@medisin.uio.no, Ellen Ruud: elruud@ous-hf.no, Hanne Cathrine Lie: \\ h.c.lie@medisin.uio.no.
}

Key words: late effects, follow-up care, clinical communication, survivorship

Corresponding Author:

Anneli Mellblom

Department of Behavioural Sciences in Medicine

POB 1111 Blindern

0317 Oslo, Norway

$\mathrm{Ph}:+4722851115$

Email: anneli.mellblom@medisin.uio.no 


\section{Abstract \\ Objective}

To explore whether negative emotions expressed by adolescent cancer survivors during follow-up consultations were associated with potential late effects (persisting disease or treatment-related health problems).

\section{Methods}

We video-recorded 66 follow-up consultations between 10 pediatric oncologists and 66 adolescent survivors of leukemia, lymphoma or stem-cell transplantations. In transcripts of the recordings, we identified utterances coded as both 1) expressions of negative emotions (VR-CoDES), and 2) late effect-related discussions. Principles of thematic content analysis were used to investigate associations between the two.

\section{Results}

Of the 66 video-recorded consultations, 22 consultations contained 56 (49\%) utterances coded as both emotional concerns and discussions of potential late effects. Negative emotions were most commonly associated with late effects such as fatigue ("I'm struggling with not having energy"), psychosocial distress ("When I touch this (scar) I become nauseous"), pain ("I'm wondering how long I am going to have this pain?”), and treatment-related effects on physical appearance (“Am I growing?”).

\section{Conclusions}

Negative emotions expressed by adolescent cancer survivors during follow-up consultations were frequently associated with potential late effects. These late effects were not the medically most serious ones, but reflected issues affecting the adolescents' daily life.

\section{Practice implication}

Eliciting and exploring patients' emotional concerns serve as means to obtain clinically relevant information regarding potential late effect and to provide emotional support. 


\section{Introduction}

The growing population of childhood cancer survivors face many challenges after treatment completion, including risk for disease- and treatment-related health problems (late effects), such as cardiorespiratory disorders, endocrine dysfunction, secondary cancers, neurocognitive impairments and fatigue [1-5]. Late effects can appear during or immediately after cancer therapy or up to decades after end of treatment, potentially interfering with physical, psychological and social functioning and well-being [6]. Up to $80 \%$ of childhood cancer survivors develop a serious or lifethreatening late effect by the age of 45 [4]. A subgroup of survivors also struggles with ongoing psychosocial problems following the cancer experience, with increased levels of anxiety and depression compared to healthy peers [6-9].

Childhood cancer survivors typically attend follow-up care 5-10 years after treatment completion. Aims of follow-up care are to detect recurrence, and at later stages detect and manage late effects, as well as educating patients about their health risks and future health care needs $[4,10,11]$. Clinicians may be reluctant to burden their patients with information about risks of late effects to avoid causing concerns about late effects that may never arise [12], while survivors have expressed ambivalent feelings towards receiving such information [13]. Yet, information about late effects is one of the most commonly reported unmet information needs among childhood cancer survivors [13].

We have previously investigated to what extent information about late effects is provided [14] and the expression of emotional concerns [15] during follow-up consultations with adolescent survivors of childhood cancers and pediatricians. We found that a vast majority of the consultations contained discussions about late effects and half of the consultations contained expressions of emotional concerns, with cancer-related concerns being one of the most common themes.

To what extent discussions of potential late effects were associated with expressed emotional concerns were, however, not explored. Knowledge of potential relationships between late effects related issues and patients' expressions of emotional concerns would be helpful for clinicians to both adapt information provided about late effects and to serve as an indication of which late effect are of particular concern for young survivors.

In this article, we perform a secondary analysis to examine 1) to what extent the expressions of emotional concerns are associated with (potential) late effects, and 
2) which late effects are most often associated with expressions of emotional concerns during follow-up consultations.

\section{Methods}

\subsection{Sample and procedures}

The sample consists of video-recordings of 66 routine follow-up consultations between 66 adolescent childhood cancer survivors and 10 pediatricians at the pediatric oncology department at Oslo University Hospital in Norway 2010-2012. One or both parents were present in $87 \%$ of the consultations. The follow-up consultations were typically scheduled for 30 minutes. Patients treated for leukemia, lymphomas or who had received a stem-cell transplant preceded by high dose chemotherapy treatment were consecutively recruited. Invitations were included with the consultation appointment letter from the hospital. A small video-recorder was placed in the corner of the consultation room and the video-recordings were transcribed verbatim. Of 88 patients invited, 70 consented to participate, and 66 had resulting video-recordings that could be analyzed (further details are provided in [15]).

\subsection{Previous analyses of expressions of emotional concerns and discussions of} (potential) late effects

Expressions of emotional concerns by the patients or the parents were identified using the Verona Coding Definitions of Emotional Sequences (VR-CoDES) [16,17]. The VR-CoDES is a consensus-based coding system that distinguishes between emotional concerns expressed as "cues", a vague, implicit expression of emotion, and a “concern", an unambiguous, explicit expression of a negative emotion [17]. Expressions of cues and concerns can be elicited spontaneously by the patient/parent or in response to a question or utterance by the physician. Half of the patients expressed at least one emotional concern, and 10\% expressed many (>7).

Communication about potential late effects was identified using a list of topics and symptoms reflecting late effects compiled by a pediatric oncologist. Patient, parent and pediatricians' utterances containing these topics or symptoms were coded accordingly, regardless of who initiated the topic. The pediatrician's utterance would be coded as communication about potential late effect regardless of what the patient replies (for more details see [14]). Communication about late effects was identified in $85 \%$ of the consultations [14]. 


\subsection{Analysis for associations between expressions of emotional concerns and}

discussions of potential late effects

We adapted principles of thematic content analysis [18] to investigate associations between emotional concerns and potential late effects-related discussions. First, we identified consultations containing expressions of emotional concerns. Second, in these, we examined each utterance coded as emotional concern or as potential late effects and extracted those coded as both. Third, the utterances were coded for the theme of the emotional concern expressed. Themes were then grouped according to overarching themes of potential late effects.

\section{Results}

Patients' characteristics are shown in Table 1. In brief, about two-thirds of the sample was female, with an average age of 15 years, on average six years from diagnosis, with leukemia being the most common diagnosis (Table 1).

Table 1. Characteristics of the total sample of adolescent cancer survivors.

\begin{tabular}{lc}
\hline Patients characteristics & $\begin{array}{c}\text { Patients included (n=66) } \\
\text { Mean (SD, range) }\end{array}$ \\
\hline Current age & $15.3(2.3,12-20)$ \\
Age at diagnosis in years & $6.7(4.0,0-16)$ \\
Years since treatment & $6.3(3.4,0-16)$ \\
Gender & Number (\%) \\
Female & $45(68)$ \\
Diagnoses & \\
Leukemia & $47(71)$ \\
Lymphoma & $14(21)$ \\
SCT for benign disorder & $5(8)$ \\
Late effect risk ${ }^{1}$ level 2 & $44(67)$ \\
Late effect risk level 3 & $22(33)$
\end{tabular}

Note. ${ }^{1}$ Patients' risk of late effects was calculated according to Oeffinger et. al. criteria [19]. Level 2: low to moderate doses of alkylating agent, anthracycline, bleomycin, or epipodophyllotoxin and no radiation. Level 3: high doses, radiation and/or stem cell transplant. 
Of the 66 video-recorded consultations, 33 contained expressions of in total 114 emotional concerns. Of these, 22 consultations contained 56 (49\%) utterances coded as both emotional concerns and discussions of potential late effects (41 expressed by the patients and 15 by a parent), and thus formed the basis for further analyses (Figure 1).

\section{Total sample: \\ 66 video-recorded consultations}

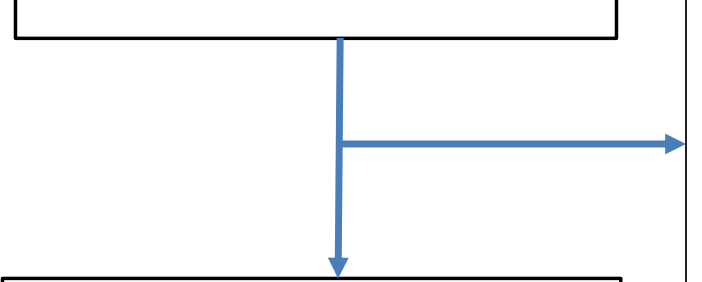

33 video-recorded consultations contained expressions of 114 emotional concerns
Excluded consultations $(n=33)$ :

- 28 contained late effects discussions but no expressions of emotional concerns

- 5 contained neither emotional concerns nor discussions of late effects
Excluded consultations ( $n=11)$ :

- 5 consultations contained emotional concerns but NO Late effects

- 6 consultations contained emotional concerns and late effects utterances, but the respective utterances did not overlap

22 consultations containing 56 overlapping utterances of expressions of emotional concerns and discussions of late effects (late effects-related emotional concerns)

Figure 1: Flow chart over number of consultations containing discussions of expressed emotional concerns and late effects issues

Seventeen of these patients were female and four patients (all female) were alone with their pediatrician. Twelve patients expressed 1 cue/concern, 6 expressed 2-4 cue/concerns and 4 expressed 7 cues/concerns.

\subsection{Associations between emotional concerns and late effects}

The themes of the late effects-related emotional concerns expressed are presented in Table 2. Fatigue was the potential late effect discussed the most frequently during the consultations and was the most common theme of late-effects related emotional concerns (e.g. "I'm struggling with not having any energy", female 
18 years). The second most frequently expressed late effects-related emotional concern was psychological distress, encompassing sub-themes such as depressive symptoms and anxiety (e.g. I think that I soon... You have to hit the bottom before it can get better, female 19 years old). Other common late effects-related emotional concerns were pain (e.g. "I'm wondering, how long am I going to have this pain?", female 17 years old), treatment-related change of physical appearance ("Do I grow, is my height normal?", male 13 years old) and school-related issues (“I don't understand anything in school (cries)", female 15 years old).

Table 2. Type and frequency of potential late effects discussed and coded as emotional concerns.

\begin{tabular}{lll}
\hline Potential late effect & $\begin{array}{l}\text { Number of times } \\
\text { raised, but not coded } \\
\text { as emotional concern }\end{array}$ & $\begin{array}{l}\text { Number of times raised } \\
\text { and coded as emotional } \\
\text { concern, n, (\%) }\end{array}$ \\
\hline Fatigue & 15 & $12(44 \%)$ \\
Psychosocial distress ${ }^{b}$ & 3 & $10(77 \%)$ \\
Pain & 6 & $7(54 \%)$ \\
Treatment-related change of & 0 & $7(100 \%)$ \\
physical appearance & & \\
School-related & 10 & $6(38 \%)$ \\
difficulties/Learning & & $3(17 \%)$ \\
difficulties & 15 & $2(50 \%)$ \\
Neuropathy, epilepsy & 2 & $2(12 \%)$ \\
Sleep problems & 15 & $2(11 \%)$ \\
Diet & 16 & $2(25 \%)$ \\
Fertility & 6 & $1(5 \%)$ \\
Cognitive problems & 20 & $1(8 \%)$ \\
Endocrinopathy & 12 & $1(100 \%)$ \\
Cardiovascular complications & 0 & $56(32 \%)$ \\
Loss of hearing & 120 & \\
\hline Total & & \\
\hline Notes. ${ }^{a} \%$ of the number of times that potential late effect was discussed and was the topic of an \\
emotional concern.
\end{tabular}

Discussions of the potentially more serious or even life-threatening late effects such as cardiac disease, endocrinopathy or fertility were seldom associated with expressions of emotional concerns, but focused mostly on practical aspects (e.g. screening attendance). Emotional concerns not associated with late effects were often 
associated with other cancer-related topics e.g. attending the follow-up consultation as previously described [15].

\section{Discussion and conclusion}

Potential late effects appeared to be a significant cause for concern among adolescent survivors of childhood cancer in this observational study of follow-up consultations. About half of the utterances coded as expressions of emotional concerns were also coded as discussions of potential late effects. Of these, the majority $(75 \%)$ did not reflect serious medical late effects, but rather those likely to interfere with psychological, social or school functioning (e.g. fatigue, psychosocial distress, pain, changes in physical appearance and school difficulties). The most serious medical late effects (e.g. fertility issues, cardiac disease, neuropathy, epilepsy and endocrinopathy), were often discussed during the consultations, but were seldom related to expressions of emotional concerns.

The literature on the nature of worries of young childhood cancer survivors is limited. Questionnaire-based studies report that survivors worry about a wide range of issues like fertility, risk for future health problems including relapse or new cancer [20-22]. These topics were rather infrequently associated with the emotional concerns expressed in this study. These differences may reflect the research methods used, answering predefined themes of potential worry versus analyzing emotional concerns expressed naturally during actual follow-up consultations. Moreover, many medical late effects are often asymptomatic at the time of follow-up care, thus representing future health risks. Given the survivors' young age it is likely that the "here and now" is perceived as more relevant or important compared to something that might happen in the future [23]. Lastly, the parents were present in almost all consultations. This may hamper the adolescents in disclosing their concerns to the physician in order to protect their parents from further worries [24].

Limitations of the study are that we do not know to what extent the expressed emotional concerns reflect the actual concerns of the patients. Interviews of the patients could have been used to validate our findings. Moreover, the small sample size also limits the generalizability of our findings. However, this study represents to our knowledge the first investigation of associations between naturally occurring expression of emotional concerns and potential late effects among cancer survivors during follow-up care visits. It supports the usefulness of combing research methods 
to build our understanding of the importance of emotional communication in cancer care.

\subsection{Conclusion}

Emotional concerns expressed at follow-ups after childhood cancer were frequently related to potential late effects, such as fatigue, pain, physical appearance and schoolrelated challenges. These emotional concerns reflect issues particularly relevant to adolescents, affecting many aspects of their everyday life.

\subsection{Practice implications}

By being sensitive to patient's expression of emotional concerns, the physician can both provide emotional support and explore more thoroughly issues related to late effects after cancer treatment. This will in turn allow physicians to provide more targeted information and guidance regarding possible medical or social interventions.

\section{References}

[1] Oeffinger KC, Mertens AC, Sklar CA, Kawashima T, Hudson MM, Meadows AT, et al. Chronic Health Conditions in Adult Survivors of Childhood Cancer. N Engl J Med. 2006;355:1572-82.

[2] Armstrong GT, Liu Q, Yasui Y, Huang S, Ness KK, Leisenring W, et al. Longterm outcomes among adult survivors of childhood central nervous system malignancies in the Childhood Cancer Survivor Study. J. Natl. Cancer Inst. 2009;101:946-58.

[3] Reulen RC, Frobisher C, Winter DL, Kelly J, Lancashire ER, Stiller CA, et al. Long-term risks of subsequent primary neoplasms among survivors of childhood cancer. J Amer Med Assoc. 2011;305:2311.

[4] Hudson MM, Ness KK, Gurney JG, Mulrooney DA, Chemaitilly W, Krull KR, et al. Clinical Ascertainment of Health Outcomes Among Adults Treated for Childhood Cancer Outcomes Among Adult Survivors of Childhood Cancer. J Amer Med Assoc. 2013;309:2371-81.

[5] Kero AE, Järvelä LS, Arola M, Malila N, Madanat-Harjuoja LM, Matomäki J, et al. Late mortality among 5-year survivors of early onset cancer: A population-based register study. Int. J. Cancer. 2014. 
[6] Zebrack BJ, Zeltzer LK, Whitton J, Mertens AC, Odom L, Berkow R, et al. Psychological outcomes in long-term survivors of childhood leukemia, Hodgkin's disease, and non-Hodgkin's lymphoma: a report from the Childhood Cancer Survivor Study. Pediatrics. 2002;110:42-52.

[7] Brinkman TM, Zhu L, Zeltzer LK, Recklitis CJ, Kimberg C, Zhang N, et al. Longitudinal patterns of psychological distress in adult survivors of childhood cancer. Br. J. Cancer. 2013;109:1373-81.

[8] Zeltzer LK, Recklitis C, Buchbinder D, Zebrack B, Casillas J, Tsao JC, et al. Psychological status in childhood cancer survivors: a report from the Childhood Cancer Survivor Study. J. Clin. Oncol.. 2009;27:2396-404.

[9] Kazak AE, DeRosa BW, Schwartz LA, Hobbie W, Carlson C, Ittenbach RF, et al. Psychological outcomes and health beliefs in adolescent and young adult survivors of childhood cancer and controls. J. Clin. Oncol. 2010;28:2002-7.

[10] Oeffinger KC, McCabe MS. Models for delivering survivorship care. J. Clin. Oncol. 2006;24:5117-24.

[11] Skinner R, Wallace WHB, Levitt G. Long-term follow-up of children treated for cancer: why is it necessary, by whom, where and how? Arch. Dis. Child. 2007;92:257-60.

[12] Cox A, Faithfull S. 'They're survivors physically but we want them to survive mentally as well': health care professionals' views on providing potential late effect information. Support Care Cancer 2013:21(9):2491-2497.

[13] Lie HC, Loge JH, Fosså SD, et al. Providing information about late effects after childhood cancer: Lymphoma survivors' preferences for what, how and when. Patient Education and Counseling 2015:98(5):604-611.

[14] Mellblom AV, Korsvold L, Finset A, Loge J, Ruud E, Lie HC. Providing Information About Late Effects During Routine Follow-Up Consultations Between Pediatric Oncologists and Adolescent Survivors: A Video-Based, Observational Study. J Adolesc Young Adult Oncol. 2015.

[15] Mellblom AV, Finset A, Korsvold L, Loge JH, Ruud E, Lie HC. Emotional concerns in follow-up consultations between paediatric oncologists and adolescent survivors: a video-based observational study. Psychooncology. 2014;23:1365-72. [16] Del Piccolo L, de Haes H, Heaven C, Jansen J, Verheul W, Bensing J, et al. Development of the Verona coding definitions of emotional sequences to code health 
providers' responses (VR-CoDES-P) to patient cues and concerns. Patient Educ Couns. 2011;82:149-55.

[17] Zimmermann C, Del Piccolo L, Bensing J, Bergvik S, De Haes H, Eide H, et al. Coding patient emotional cues and concerns in medical consultations: The verona coding definitions of emotional sequences (VR-CoDES). Patient Educ Couns. 2011;82:141-8.

[18] Guest G, MacQueen KM, Namey EE, Applied thematic analysis, SAGE Publications, Incorporated, Thousand Oaks, CA, 2011.

[19] Oeffinger KC, Nathan PC, Kremer LCM. Challenges after curative treatment for childhood cancer and long-term follow up of survivors.

Pediatr. Clin. North Am. 2008;55:251-73.

[20] Wang R, Syed IA, Nathan PC, Barr RD, Rosenberg-Yunger ZR, Klassen AF. Exploring Cancer Worry in Adolescent and Young Adult Survivors of Childhood Cancers. J Adolesc Young Adult Oncol. 2015.

[21] Zebrack BJ, Chesler M. Health-related worries, self-image, and life outlooks of long-term survivors of childhood cancer. Health Soc Work. 2001;26:245-56.

[22] Langeveld NE, Grootenhuis MA, Voute PA, de Haan RJ, van den Bos C. Quality of life, self-esteem and worries in young adult survivors of childhood cancer. Psychooncology. 2004;13:867-81.

[23] Christie D, Viner R. Adolescent development. Brit Med J. 2005;330:301-4.

[24] Kent EE, Parry C, Montoya MJ, Sender LS, Morris RA, Anton-Culver H. "You're too young for this": Adolescent and Young Adults' Perspectives on Cancer Survivorship. J Psychosoc Oncol. 2012;30:260-79. 